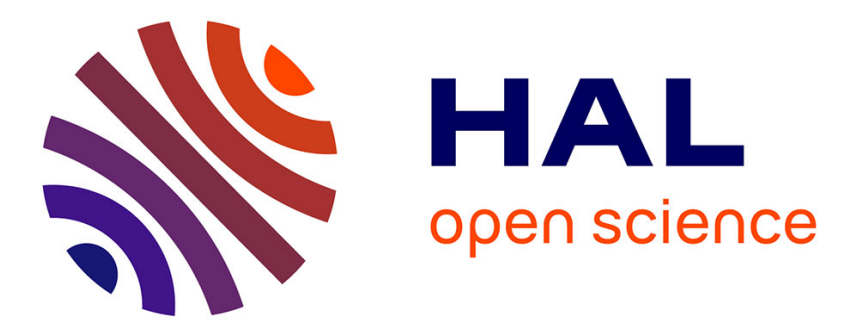

\title{
Towards a Reliable Vision-Based Mobile Robot Formation Control
}

Pierre Renaud, Enric Cervera, Philippe Martinet

\section{To cite this version:}

Pierre Renaud, Enric Cervera, Philippe Martinet. Towards a Reliable Vision-Based Mobile Robot Formation Control. IROS: Intelligent RObots and Systems, Sep 2004, Sendai, Japan. pp.3176-3181, 10.1109/IROS.2004.1389906 . lirmm-00108919

\section{HAL Id: lirmm-00108919 https://hal-lirmm.ccsd.cnrs.fr/lirmm-00108919}

Submitted on 4 May 2016

HAL is a multi-disciplinary open access archive for the deposit and dissemination of scientific research documents, whether they are published or not. The documents may come from teaching and research institutions in France or abroad, or from public or private research centers.
L'archive ouverte pluridisciplinaire HAL, est destinée au dépôt et à la diffusion de documents scientifiques de niveau recherche, publiés ou non, émanant des établissements d'enseignement et de recherche français ou étrangers, des laboratoires publics ou privés. 


\section{Towards a reliable vision-based mobile robot formation control}

\author{
Pierre Renaud \\ LIRMM \\ Montpellier, France \\ renaud@lirmm.fr
}

\author{
Enric Cervera \\ Robotic Intelligence Laboratory \\ Castellon, Spain \\ ecervera@icc.uji.es
}

\author{
Philippe Martinet \\ LASMEA \\ Clermont-Ferrand, France \\ martinet@lasmea.univ-bclermont.fr
}

\begin{abstract}
In this article, a robot formation control strategy based on a vision-based follow-the-leader scenario is proposed, with emphasize on its reliability. On the one hand, perception is enhanced by the control of a motorized zoom. On the other hand, bidirectional and non-oblivious [1] control is implemented, with an odometry-based fault detection of vision-based information and a leader path-planning strategy to improve its visibility. After introduction of the control strategy, extensive experimental results are presented.
\end{abstract}

\section{INTRODUCTION}

Control of robot formations has received much attention these past years. Indeed, the use of several coordinated mobile robots enables one to achieve complex tasks [2], [3], [4], with a low robot complexity, since each one can be specialized in one task such as navigation or manipulation. The previously proposed control strategies offer different degrees of robustness of the formation, i.e. reliability with respect to real world conditions. Perception, formation and control characteristics, the three main criteria cited in [5] to classify the robot formation control strategy, influence this robustness, as shown in the following.

Using the robot proprioceptive sensors and inter-robot communication, self-localization can be achieved [6]. Odometry accuracy remains however delicate [7]. Dead reckoning is then limited, and will still fail in case of wheel slippage or contact with an obstacle. Vision has been therefore proposed to get an exteroceptive information. With omnidirectional cameras [8] a $360^{\circ}$ detection is possible, within however a limited distance (in the order of $1.5 \mathrm{~m}$ in [9]). Due to the large field of view, the accuracy may also be limited if a standard camera is used. Directional cameras have complementary performances, with visibility constraints [5], at however a much lower cost [10]. Concerning the image processing, color has been widely used to achieve the robots detection [11], [5], [12]. The robustness of color detection with respect to light conditions can however be a major source of detection failure [13]. Eventually, a global exteroceptive measurement [14] is difficult to achieve in indoor applications.

Formation characteristics can improve its reliability. In a unit-referenced formation [14], or point-referenced with the use of a virtual structure [15], the perception by each robot of the other members enables one to get redundancy which increases the formation robustness. The need for a complete formation perception is however very restricting.
With a neighbor-referenced approach, if the link between the robots is unidirectional [16], the follower navigation is on the contrary completely dependent on the perception of its neighbors. A neighbor failure cannot be detected and therefore compensated. At the opposite, in the bidirectional formation control proposed in [11], the failure of one robot is detected by the interruption of the emission of its identification number. Like in [5], the proposed robot attitude is the re-formation of the robots, which is however not adequate with a local loss of information of one robot, because of a vision failure or a temporary occlusion.

Eventually, the ability in the control to manage obstacle avoidance, change of formation geometry can improve the reliability of the formation in unknown environments. The use of a non-oblivious [1] control scheme, i.e. with a behavior dependent on the past information, is also likely to improve the robustness of the control scheme.

We have proposed in [17] a simple and efficient way to control robot formations. Leader-follower control is used, with unidirectional control, using vision-based leader pose estimation. In this article, we develop the reliability of this formation control. On the one hand, the perception is enhanced by the control of a motorized zoom. Leader pose estimation is hence possible in a larger field-of-view, with higher accuracy. On the other hand a bidirectional and non-oblivious control strategy is implemented to improve the reliability of the vision-based scheme: an odometrybased fault-detection is used to detect vision-based pose estimation failure and a novel path-planning scheme is proposed to control the leader and avoid loss of visibility by the follower.

In the second section, the follow-the-leader control scheme is recalled with the description of the development platform. In the third section, the implementation of the zoom control and its advantages are then outlined, before in the fourth section introducing the control strategy proposed to improve the reliability of the vision-based follow-theleader. Extensive experimental results are then given in the fifth section, before concluding on the performance of the robots control and further developments.

\section{THE ROBOT FORMATION CONTROL SCENARIO}

\section{A. Robot formation control}

The control of the formation is using a decentralized algorithm. The leader of the formation is considered spe- 
cialized in the navigation task, using for instance a laser to build environment maps and achieve the localization task. Each follower robot measures the pose (i.e. position and orientation) of its leader using vision. The follower trajectory is computed by considering a Bezier curve [17] between the follower robot current position and the estimated leader relative position.

For the considered unicycle robot model, the Bezier curve allows us to compute trajectories wich are tangent to current leader and follower paths. Assuming the needed angular velocity can be obtained with the follower robot, the computed path is compatible with the nonholonomic constraint. This assumption is generally verified experimentally. Furthermore, the follower is then able to avoid collisions with obstacles bypassed by the leader by properly designing the Bezier curve (figure 2). Finally, formations can be controlled by simply defining virtual leader positions from the current one (figure 2).

\section{B. Hardware platform}

Commercial mobile robots Pioneer 2 [18] (figure 3) are used for our application. All the robots are equipped with sonars for obstacle detection and wireless communication (IEEE 802.11b). Control of each robot is performed using an embedded PC.

The leader is equipped with a laser to build environment maps and achieve the localization task. A calibration board is mounted on it, and the follower is equipped with a pan-tilt-zoom camera (Canon VCC4, 4-64mm zoom) to observe its leader. The calibration board is composed of three different colored areas, which are detected using a blob detector. The pose of the leader robot $(d, \alpha)$ (figure 1) can hence be determined [17].

\section{Software platform}

The formation control is developed in Acromovi [19], a framework specially designed for the development of

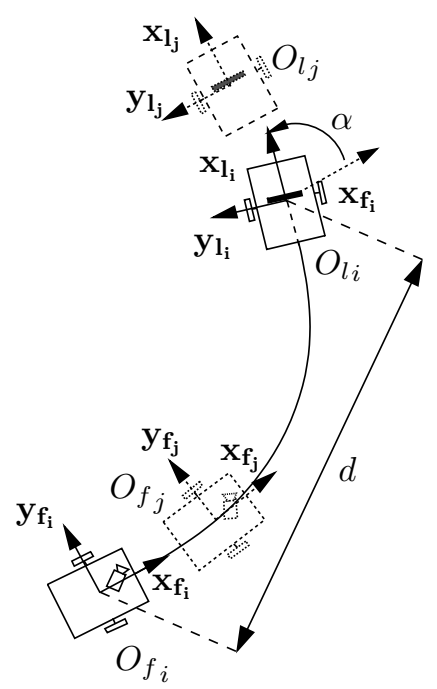

Fig. 1. The leader and follower at two time instants $i$ and $j$, with the Bezier trajectory determined from their positions at time instant $i$.
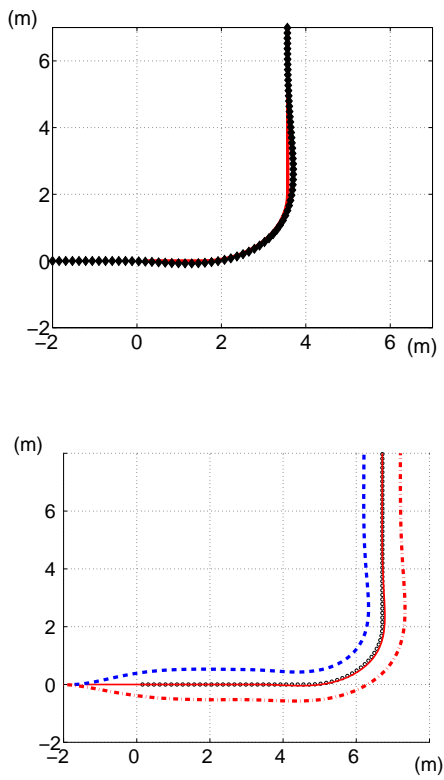

Fig. 2. Obstacle bypass simulation $\left(90^{\circ}\right.$ turn, radius $2 m$, speed: $0.15 \mathrm{~m} / \mathrm{s}$, distance leader/follower: $2 \mathrm{~m}$, time increment: $1 \mathrm{~s})$. On the top with one follower, on the bottom with three followers.

distributed applications for a team of heterogeneous mobile robots. The software architecture gives us the ease of development of cooperative tasks among robots, using an agent-based platform. In particular, communication between robots can be easily integrated to the control scheme.

\section{Perception enhancement}

\section{A. Pan-Tilt-Zoom control}

In this section, we propose to use a motorized zoom to lower the detection failure of the calibration board and improve the pose estimation accuracy. The use of zoom has been proposed in the context of active vision [20] or visual servoing, with explicit knowledge of intrinsic parameters from calibration [21], [22], or not [23]. In our context, the communication protocol between the robot and the camera limits the camera control frequency $(\sim 1 \mathrm{~Hz})$, hence restricting the vision-based camera control to a static look and move strategy [24]. Camera intrinsic parameters are

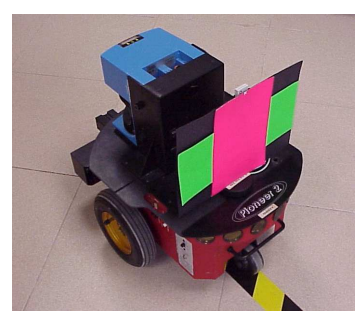

(a) Leader robot

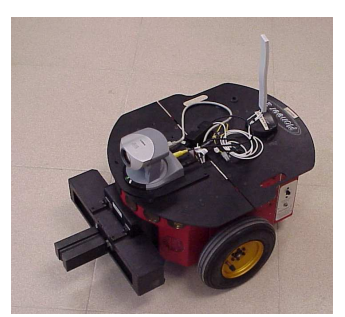

(b) Follower robot
Fig. 3. Leader and follower robots. 


\section{TABLE I}

ESTIMATED STANDARD DEVIATIONS OF THE LEADER POSE MEASUREMENTS. CROSSES $(\times)$ FOR DETECTION FAILURE OF THE CALIBRATION BOARD. IN ITALIC: WITHOUT ZOOM, IN BOLD: WITH ZOOM. VALUES ROUNDED TO $0.01 \mathrm{M}, 0.01^{\circ}$

\begin{tabular}{|c|c|c|}
\hline$d(m)$ & $4\left(^{\circ}\right)$ & $65^{\circ}$ \\
\hline $1 m$ & $0.01 m / \mathbf{0 . 0 1 m}$ & $0.01 m / \mathbf{0 . 0 1 m}$ \\
& $0.6^{\circ} / \mathbf{0 . 6}$ & $0.3^{\circ} / \mathbf{0 . 3}$ \\
\hline \multirow{2}{*}{$2 m$} & $0.05 m / \mathbf{0 . 0 2}$ & $0.02 m / \mathbf{0 . 0 1 m}$ \\
& $25^{\circ} / \mathbf{1}^{\circ}$ & $0.3^{\circ} / \mathbf{0 . 3}$ \\
\hline \multirow{2}{*}{$3 m$} & $\times / \mathbf{0 . 0 3 m}$ & $\times / \mathbf{0 . 0 4 m}$ \\
$\times / \mathbf{0 . 6}^{\circ}$ & $\times / \mathbf{0 . 6}^{\circ}$ \\
\hline
\end{tabular}

identified during a prior calibration.

The calibration board center and the height of the center color card in the image are used to compute the pan and zoom corrections to apply to the camera. Since the calibration board center is in our context at the same height that the camera, no tilt correction is needed. Let $\left(f, \Delta o_{l}, h\right)$ be respectively the current focal length, gap in the image between the camera image center and the calibration board center in the horizontal direction and the size in the image of the central element of the calibration board. The pan and zoom corrections $(\Delta P, \Delta f)$ to apply at each time step are then computed by first order approximation as:

$$
\left\{\begin{aligned}
\Delta P & =\frac{\Delta o_{l}}{f} \\
\Delta f & =\left(\frac{h_{\text {des }}}{h}-1\right) \cdot f
\end{aligned}\right.
$$

with $h_{\text {des }}$ the desired size in the image of the calibration board central element.

\section{B. Simulation results}

The improvement of the pose estimation accuracy has been evaluated experimentally through repeatability measurements in different configurations. In table I the standard deviations of the calibration board angle and distance are indicated for three distinct distances. The fronto-parallel position $(\alpha=0)$ is not considered because for small values the angle is set to zero.

The use of the zoom improves the ability to detect the calibration board and the accuracy. A significant accuracy improvement of the follower trajectory can also be observed by simulating the trajectory of figure 2 with addition of the experimentally evaluated measurement noise (figure 4). When used, the zoom magnification is approximately equal to 2 . Without zoom, the gaps between leader and follower noise are up to $0.3 \mathrm{~m}$, which can cause interferences with another robot or an obstacle.

\section{Control Enhancement}

The follower robot is controlled by computing at each time step the vision-based pose estimation as mentioned in the second section, and then the corresponding Bezier path. In this section, we propose two modifications of the control scheme to improve the reliability of the follow-the-leader application. The first one is based on the redundancy of the odometry with respect to the vision-based perception. The

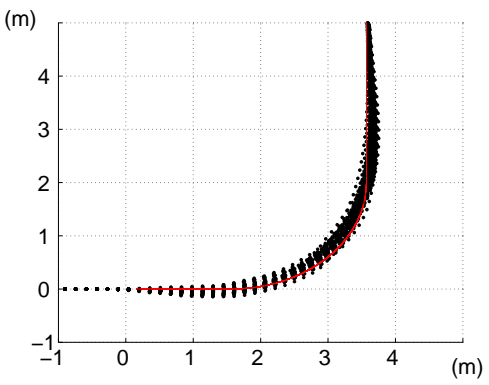

(a) Without zoom

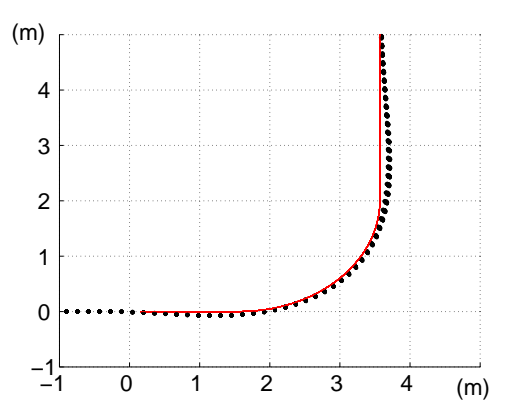

(b) With zoom

Fig. 4. Simulation of obstacle bypass using the pose measurement noise experimentally evaluated: trace of 50 computed trajectories (simulation conditions of figure 2).

second one is an original path-planning strategy to avoid the loss of the leader image by the follower.

\section{A. Leader state estimation}

Vision is an adequate sensor for the robot control, with accuracy in the order of $\left(1 \mathrm{~cm}, 1^{\circ}\right)$ for indoor applications with off-the-shelf cameras. Variable light conditions, calibration board occlusions may however significantly alter the calibration board color cards detection, leading to outliers in the vision-based pose estimation. To improve the follower control reliability, communication between follower and leader and their odometry are hence used to get a redundant leader state estimation.

For sake of simplicity, the calibration board and the camera are supposed located on the rotation axes of the robots. The position of the leader with respect to the follower at time instant $j$ from situation at instant $i$ can be predicted with the relation (figure 1):

$$
\left.O_{f_{j}} O_{l_{j}}\right|_{R_{f_{j}}}=\left.O_{f_{j}} O_{f_{i}}\right|_{R_{f_{j}}}+\left.O_{f_{i}} O_{l_{i}}\right|_{R_{f_{j}}}+\left.O_{l_{i}} O_{l_{j}}\right|_{R_{f_{j}}}
$$

with at first order:

$$
\left\{\begin{array}{l}
\left.O_{f_{j}} O_{f_{i}}\right|_{R_{f_{j}}}=-\left.v_{f_{i}} \Delta \mathrm{t} \cdot \mathbf{x}_{\mathbf{f}_{\mathbf{j}}}\right|_{R_{f_{j}}} \\
\left.O_{l_{i}} O_{l_{j}}\right|_{f_{j}}=\left.v_{l_{i}} \Delta \mathrm{t} \cdot \mathbf{x}_{\mathbf{l}_{\mathbf{i}}}\right|_{R_{f_{j}}}
\end{array}\right.
$$

where $v$ represent the current translational speed, estimated from odometry. 
From eq. (2), the pan angle of the camera can also be predicted with:

$$
\Theta_{j}=\operatorname{Atan}\left(\frac{O_{l}^{i} O_{l}^{j} \cdot y_{f_{j}}}{O_{l}^{i} O_{l}^{j} \cdot x_{f_{j}}}\right)
$$

The leader pose estimation with respect to the follower is achieved at each iteration of the control. This enables us to detect occlusions or outliers of the vision-based pose estimation by comparison between the odometrybased estimation and the vision-based one, according to the following pseudo code:

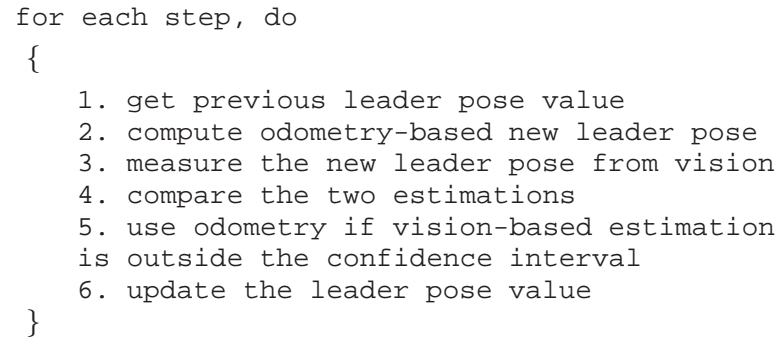

The value of the confidence interval is easily set since partial occlusion of the calibration board or color detection failure induces pose error greater than $30 \mathrm{~cm}$, to compare with the $1 \mathrm{~cm}$ vision-based pose accuracy.

Odometry accuracy bias is well known, especially for rotation measurements. It is therefore used as a redundant sensor for fault detection, but no data fusion is achieved between vision and odometry, since no such bias occurs with vision.

\section{B. Path-planning for leader visibility}

1) Leader path-planning strategy: The formation leader has the perception of its environment and its localization, considering that a previous mapping of the environment has been achieved. Several methods have been proposed to ensure the displacement of the robot to a defined goal. The gradient method proposed by in [25] present the advantages of a real-time planning taking into account the evolution of the environment, with avoidance of local minimas. In this section we introduce a modification of this planning method to ensure both the planning of the trajectory towards the goal and the vision-based control of a follower robot. The used gradient method is first briefly recalled. The reader is referred to [25] for further details.

2) The gradient method principle: The gradient method is based on the evaluation of a navigation function for each path, the optimum being the one minimizing this function. Two costs are computed at each point $p_{i}$ for its evaluation: the intrinsic $I\left(p_{i}\right)$ and adjacent $A\left(p_{i}, p_{i+1}\right)$ costs. The former represents the cost of being at the point, and the latter the cost of moving from $p_{i}$ to another point $p_{i+1}$. It is assumed that these costs are separable, so that along a path constituted by the set of points $P=\left\{p_{1}, p_{2}, \ldots\right\}$ the cost is equal to:

$$
F(P)=\sum_{p} I\left(p_{i}\right)+A\left(p_{i}, p_{i+1}\right)
$$

The navigation function at a point $p$ is defined as the minimal cost of a path between $p$ and the goal point. The

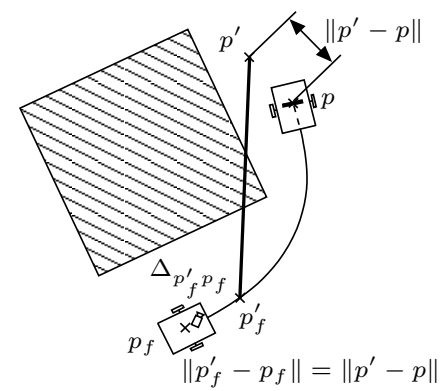

Fig. 5. Computation of the intrinsic cost bound to the visibility constraint.

robot path is hence determined by computing at each time step the costs to get the optimal path.

3) The visibility constraint: At each position $p$ of the leader (figure 5), the current position of the follower $p_{f}$ is known, either using vision or the estimation described in the previous paragraph. For any point $p^{\prime}$ around the current position of the leader, one can therefore determine the corresponding position $p_{f}^{\prime}$ of the follower and the line $\Delta_{p_{f}^{\prime} p^{\prime}}$ between the robots.

At each step, the obstacles are supposed known using the mapping and the perception with the laser and sonars. The associated intrinsic costs can then be computed, so that we can evaluate the loss of the leader image by the follower by the condition:

$$
\sum_{i} I\left(p_{i}\right)>0, \forall p_{i} \in \Delta_{p_{f}^{\prime} p^{\prime}}
$$

If the condition is fulfilled, the intrinsic cost at point $p_{i}$ is set to the cost of an obstacle. Since at each step of the leader trajectory determination the intrinsic costs are computed to determine the path, the visibility of the leader should be ensured.

The existence of a path for the leader with respect of the visibility constraint is not a priori ensured. The gradient method may therefore not find any solution to the planning of the robot leader. In such a case, the planning will be switched to the previous scheme, without considering the additional constraint.

4) Simulation results: In figure 6 two paths are generated to bring the leader robot on the other side of a wall. Without the visibility constraint, the follower loses the leader image during a significant part of the trajectory. Odometry-based trajectory estimation can then be used, but it must be assumed the absence of significant bias of the sensors. The proposed planning strategy avoids such hypothesis, with constant visibility of the leader robot.

The visibility constraint imposes the absence of obstacles between the two robots. One may notice that the angle between the two robots becomes close to $90^{\circ}$ during the leader turn on the figure 6 . The calibration board image loss can be avoided using additional calibration boards on the leader sides, or introducing an intrinsic cost linked to the value of the angle between the robots. This method will now be evaluated on our development platform. After validation, planning of a leader with several robots in 


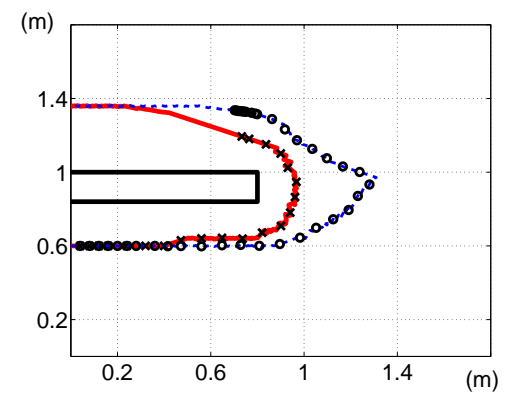

Fig. 6. Simulation of obstacle bypass planning for the leader (Final position of the leader in $(0,1.4)$, leader path without constraint in continuous line, with constraint in dotted line. Follower positions represented by crosses and circles, Distance leader/follower: $1 \mathrm{~m}$ )
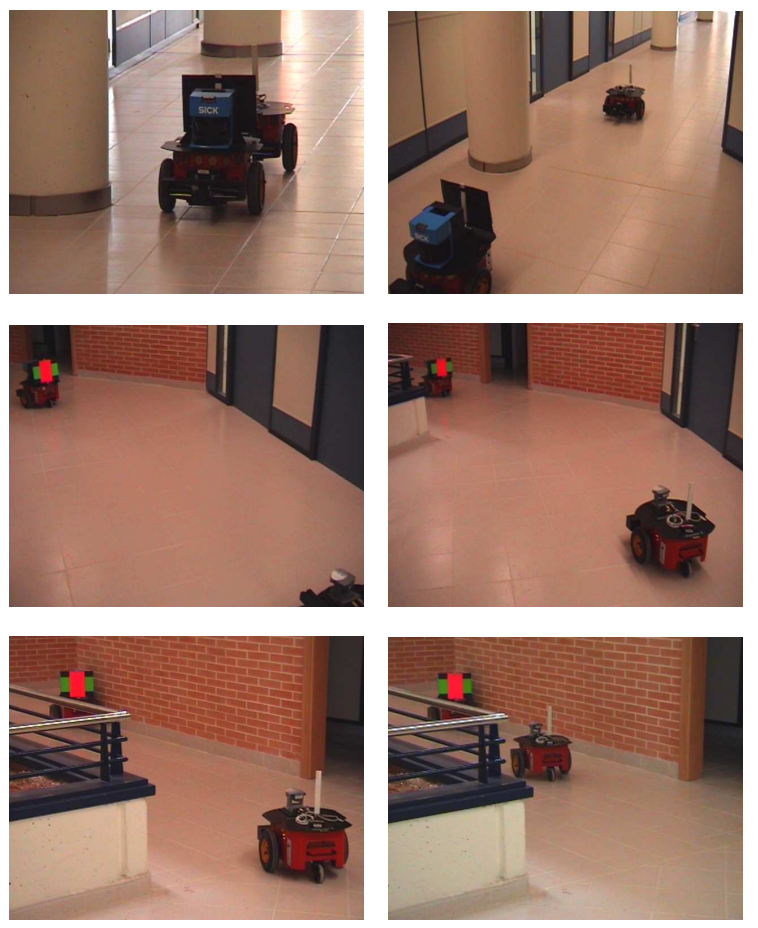

Fig. 7. Long distance following with zoom.

the formation will be implemented, as well as the angle constraint between the robots.

\section{EXPERIMENTAL RESULTS}

\section{A. Zoom contribution}

To evaluate the contribution of the zoom, in a first experiment the leader and follower are velocity controlled, with a follower having $80 \%$ of the leader speed. A $45^{\circ}$ turn is achieved by the leader at $0.2 m . s^{-1}$ (figure 7 ). The initial distance is approximately equal to $1 \mathrm{~m}$, and increases up to $5 \mathrm{~m}$ before the leader stops (figure 8).

The control of the focal length allows to use up to a $3 \times$ zoom. Without zoom the detection of the leader could not be achieved. The use of the zoom could hence enable us to design formation control with larger flexibility of the distance between the robots, and therefore on formation

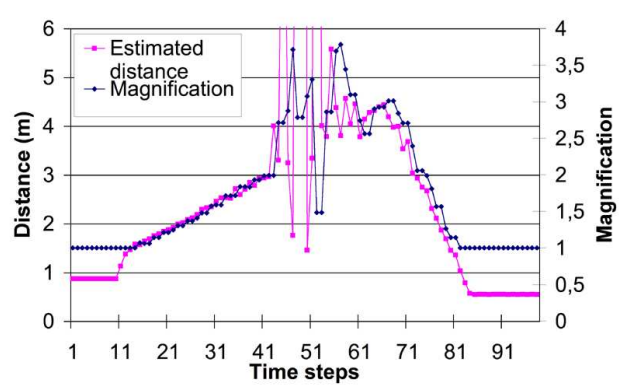

Without odometry

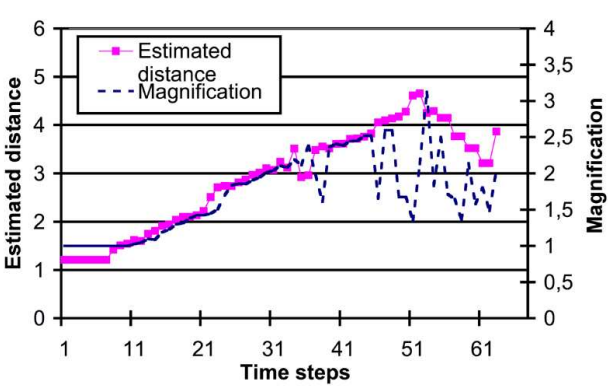

With odometry

Fig. 8. Evolution of the vision-based distance estimation and zoom magnification.

geometry. In particular, high-speed displacements may be used with large distances between the robots for safety.

The camera is programmed in that experiment to zoom out in case of detection failure. Such a failure occurs several times (figure 8), and outliers in the distance evaluation also appear. The use of odometry-based redundancy enables us to compensate these failures, as demonstrated in the next paragraph.

\section{B. Odometry-based redundancy}

1) Long distance following: With the previous experimental conditions, and the use of the odometry-based fault detection, the distance is now estimated without any outlier (figure 8).

The camera is still programmed to zoom out in case of detection failure, so that the time steps where distance estimation is achieved using odometry can be easily detected. The follower is programmed to stop simultaneously with the leader, so that this experiment is shorter than the previous one (figure 8).

2) Trajectory in the UJI building: Trajectories have been generated in the UJI Computer Science Building, with two consecutive $90^{\circ}$ from the door of the lab. This reproduces the trajectories that would be obtained by planning, if visibility constraints are not taken into account. The follow-the-leader control has been experimented using successively vision, odometry, and their combination with the previously described control scheme. The proposed strategy is the only one which enables the follower to achieve the complete trajectory (figure 9). In particular, the odometry bias (in the order of $35^{\circ}$ for a $360^{\circ}$ rotation) prevented the follower to achieve the leader path. 

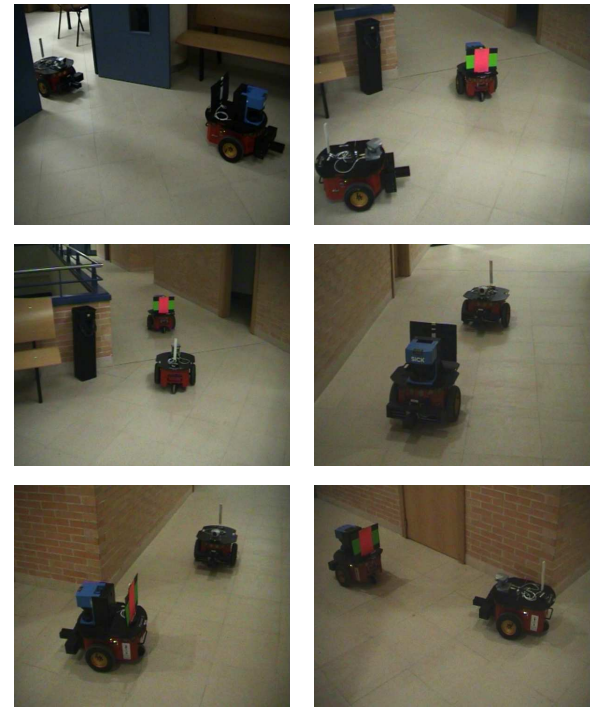

Fig. 9. Trajectory in the $U J I$ building.

\section{CONCLUSION}

In this article, a robot formation control strategy has been described, with particular developments for its reliability. On the one hand, perception is enhanced by the control a pan-tilt-zoom camera, which gives the follower robot a large field of view $\left(180^{\circ}\right.$, several meters) and improvement of the leader detection. On the other hand, bidirectional and non-oblivious robot control has been proposed, with the use of odometry to detect outliers in the follower vision-based pose estimation, and the leader path planning taking into account visibility constraint. The presented experimental results have demonstrated the improvement due to the use of zoom and odometry-based fault detection. The enhanced field of perception of the follower robot will enable us to consider new formation geometries, with higher distances between the robots, which now will be implemented. In particular, distances can be set as a function of the leader speed, to get "safe distances" between the robots. Using modified communication between camera and robot, a modified control of the camera will also be used, with a velocity-based control scheme.

\section{ACKNOWLEDGMENTS}

This research has been carried out at the Robotic Intelligence Laboratory of Universitat Jaume-I. Support for this research is provided in part by the Ministerio de Ciencia y Tecnologia under projects DPI2001-3801, HF20010112, and FIT-020100-2003-592 (PROFIT), by the Generalitat Valenciana under projects inf01-27, GV01-244, CTIDIA/2002/195, and by the Fundacio Caixa-Castello under project P1-1B2001-28. The authors gratefully acknowledge this support.

\section{REFERENCES}

[1] I. Suzuki and M. Yamashita, "Agreement on a common X-Y coordinate system by a group of mobile robots," in Proc of the Dagstuhl Seminar on Modeling and Planning for sensor-based intelligent robot systems, 1996.
[2] J. Jennings, G. Whelan, and W. Evans, "Cooperative search and rescue with a team of mobile robots," in Proc. IEEE Int. Conf. on Advanced Robotics, 1997, pp. 193-200.

[3] H. Nguyen, N. Pezehkhian, M. Raymond, A. Gupta, and J.M.Spector, "Autonomous communication relays for tactical robots," in Proc. of IEEE Int. Conf. on Advanced Robotics, Coimbra, Portugal, 2003, pp. 35-40.

[4] R. Hogg, A. Rankin, S. Roumeliotis, M. Henry, D. Helmick, C. Bergh, and L. Matthies, "Algorithms and sensors for small robot path following," in Proc. of Int Conf on Robotics and Automation, Washington DC, USA, 2002, pp. 3850-3857.

[5] F. Michaud, D. Letourneau, M. Guilbert, and J.-M. Valin, "Dynamic robot formations using directional visual perception," in Proc. of 2002 Int. Conf. on Int. Robots and Systems, Lausanne, Switzerland, october 2002, pp. 2740-2745.

[6] S. Roumeliotis and G. Bekey, "Distributed multi-robot localization," IEEE Transactions on Robotics and Automation, vol. 18, no. 5, pp. 781-795, 2002.

[7] P. Goel, S. Roumeliotis, and G. Sukhatme, "Robust localization using relative and absolute position estimates," in Proc of Int. Conf. on Int. Robots and Systems, 1999, pp. 1134-1140.

[8] A. Das, R. Fierro, V. Kumar, J. Ostrowski, J. Spletzer, and C. Taylor, "A vision-based formation control framework," IEEE Trans. on Robotics and Automation, vol. 18, no. 5, pp. 813-825, october 2002.

[9] G. Pereira, V. Kumar, and M. Campos, "Localization and tracking in robot networks," in Proc. of Int. Conf. on Advanced Robotics, Coimbra, Portugal, 2001, pp. 465-470.

[10] M. Sarcinetti-Filho, T. Bastos-Filho, and R. Freitas, "Mobile robot navigation via reference recognition based on ultrasonic sensing and monocular vision," in Proc of Int. Conf. on Advanced Robotics, Coimbra, Portugal, 2003, pp. 204-209.

[11] J. Fredslund and M. Mataric, "A general algorithm for robot formations using local sensing and minimal communication," IEEE Trans. on Robotics and Automation, vol. 18, no. 5, pp. 837-846, october 2002.

[12] A. Das, R. Fierro, V. Kumar, B. Southall, J. Spletzer, and C. Taylor, "Real-time vision-based control of a nonholonomic mobile robot," in Proc of Int. Conf. on Robotics and Automation, Seoul, Korea, 2001, pp. 1714-1719.

[13] G. D. Cubber, S. Berrabah, and H. Sahli, "A bayesian approach for color consistency based visual servoing," in Proc of Int. Conf. on Advanced Robotics, Coimbra, Portugal, 2003, pp. 983-990.

[14] T. Balch and R. Arkin, "Behavior-based formation control for multirobot teams," IEEE Transactions on Robotics and Automation, vol. 14, no. 6, pp. 926-939, 1998.

[15] M. A. Lewis an K. Tan, "High precision formation control of mobile robots using virtual coordinates," Autonomous Robots, vol. 4, pp. 387-403, 1997

[16] P. Tabuada, G. Pappas, and P. Lima, "Feasible formations of multiagent systems," in Proc of American Control Conference, Arlington, USA, 2001, pp. 56-61.

[17] S. Chiem and E. Cervera, "Vision-based robot formations with Bezier trajectories," in Proc of Intelligent and Autonomous Robots 8, 2004, pp. 191-198.

[18] "www.activmedia.com."

[19] P. Nebot, D. Gomez, and E. Cerver, "Agents for cooperative heterogeneous mobile robotics," in Proc of IEEE Int. Conf. on Syst, Man and Cybernetics, Washington, USA, 2003, pp. 557-562.

[20] R. Atienza and A. Zelinsky, "A practical zoom camera calibration technique: an application of active vision for human-robot interaction," in Proc. of Australian Conf. on Robotics and Automation, 2001, pp. 85-90.

[21] K. Hosoda, H. Moriyama, and M. Asada, "Visual servoing utilizing zoom mechanism," in Proc of Int. Conf. on Robotics and Automation, 1995, pp. 178-183.

[22] X. Clady, F. Collange, F. Jurie, and P. Martinet, "Objet tracking with a pan tilt zoom camera, application to car driving assistance," in Proc. of Int. Conf. on Robotics and Automation, Seoul, Korea, 2001, pp. 1653-1658.

[23] E. Malis, "Visual servoing invariant to changes in camera intrinsic parameters," in Proc of Int. Conf. in Computer Vision, Vancouver, Canada, 2001, pp. 704-709.

[24] A. Sanderson and L. Weiss, Robot vision. I.F.S Publications Ltd., 1983.

[25] K. Konolige, "A gradient method for realtime robot control," in Proc Int. Conf. on Intelligent Robots and Systems, 2000. 